\title{
Upgrades to the LLNL Flash X-ray Induction Linear Accelerator (FXR)
}

R. D. Scarpetti, N. L. Back, J. K. Boyd, G. G. Earley, K. L. Griffin, R. G. Kerr,

R. Kihara, M. M. Ong, J-M. Zentler

This paper was prepared for submittal to the 11th IEEE International Pulsed Power Conference

Baltimore, MD

June 29-July 2, 1997

June 30, 1997

Thivima preprint of a peper intended for publication in a joumal or proceedinge. Since changes may be made before publication, this preprint is mode avallable with the underctanding that it will not be cited oc reproduced without the perminion of the suthor. 


\section{DISCLAIMER}

This document was prepared as an account of work sponsored by an agency of the United States Government. Neither the United States Government nor the University of Callfornia nor any of their employees, makes any warranty, exprese or implied, or assumes any legal liability or respondibility for the accuracy, completeness, or usefulness of any information, apparatus, product, or procese disclosed, or represents that its use would not infringe privately owned rights. Reference herein to any specific commencial product, process, or service by trade name, trademark, manufacturer, or otherwise, does not necessarily constitute or imply its endorsement, recommendation, or favoring by the United States Government or the University of California. The views and opinions of authors expressed herein do not necessarily state or reflect those of the United States Government or the University of California, and shall not be used for advertising or product endorsement purposes. 


\title{
UPGRADES TO THE LLNL FLASH X-RAY INDUCTION LINEAR ACCELERATOR (FXR)
}

\author{
Raymond D. Scarpetti \\ Norman L. Back \\ John K. Boyd \\ Gregory G. Earley \\ Kenneth L. Griffin \\ Roderick G. Kerr \\ Ronald Kihara \\ Mike M. Ong \\ Jan-Mark Zentler \\ Lawrence Livermore National Laboratory \\ Livermore, CA
}

\begin{abstract}
The FXR is an induction linear accelerator used for flash radiography at the Lawrence Livermore National Laboratory's Site 300 Test Facility. The FXR was originally completed in 1982 and has been in continuous use as a radiographic tool. At that time the FXR produced a $17 \mathrm{MeV}, 2.2 \mathrm{kA}$ burst of electrons for a duration of $65 \mathrm{~ns}$.

An upgrade of the FXR was recently completed. The purpose of this upgrade was to improve the performance of the FXR by increasing the energy of the electron injector from $1.2 \mathrm{MeV}$ to $2.5 \mathrm{MeV}$ and the beam current from $2.2 \mathrm{kA}$ to $3 \mathrm{kA}$, improving the magnetic transport system by redesigning the solenoidal transport focus coils, reducing the rf coupling of the electron beam to the accelerator cells, and by adding additional beam diagnostics.
\end{abstract}

We will describe the injector upgrades and performance, as well as our efforts to tune the accelerator by minimizing beam corkscrew motion and the impact of Beam Breakup Instability on beam centroid motion throughout the beam line as the current is increased to $3 \mathrm{kA}$.

\section{Introduction}

The FXR 1,2 is an induction linear accelerator used for flash radiography. It is the principle diagnostic tool at the Building 801 hydrodiagnostics test bunker at the Lawrence Livermore National Laboratory's Site 300 Test Facility. The accelerator is housed in a reinforced concrete bunke and has an overall length of 42 meters (see Figure 1). It consists of eleven blocks of 4 accelerator cells each. The 44 accelerator cells each imparts an energy of from 300 to $350 \mathrm{keV}$ to the electron beam (see Figure 2). A 2.5 MV Injector generates a $65 \mathrm{ns,} 2$ to 3 kilo amperes of electron beam current which is accelerated through the 44 accelerator cells to a final energy of 16$18 \mathrm{MeV}$. This electron beam is focused onto a $1 \mathrm{~mm}$ thick tantalum target to produce an x-ray fluence (flux) of 320-400 R, one meter from the target. The result is a single radiograph of the device under test, with the $\mathrm{x}$-ray film protected in a blast-proof cassette located behind the device.

The FXR was designed and built at the Lawrence Livermore National Laboratory beginning in 1978 at a cost of approximately \$13 Million. It was activated in 1982 and since then, has been available for flash radiography. The FXR Beam Upgrade effort began in 1991 to enhance the performance of the FXR induction accelerator by, first, improving the electron beam quality by 
upgrading the electron injector and magnetic transport system and, second, by increasing the electron beam current. The result being an increase in $x$-ray fluence for greater penetrating capability and a reduction in beam spot size for improved resolution.

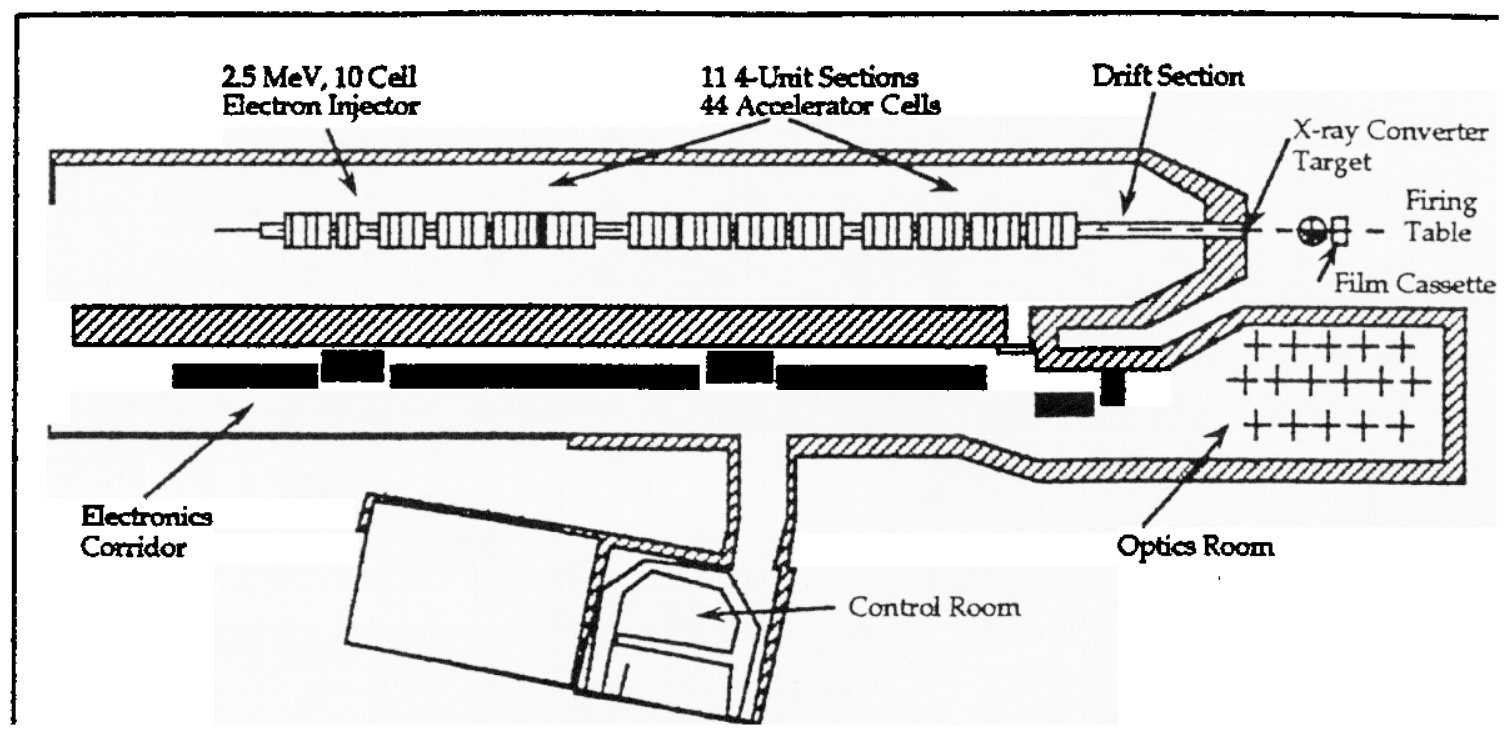

Figure 1: The FXR Linear Induction Accelerator is located in Bunker 801 at the LLNL Site 300 Test Facility

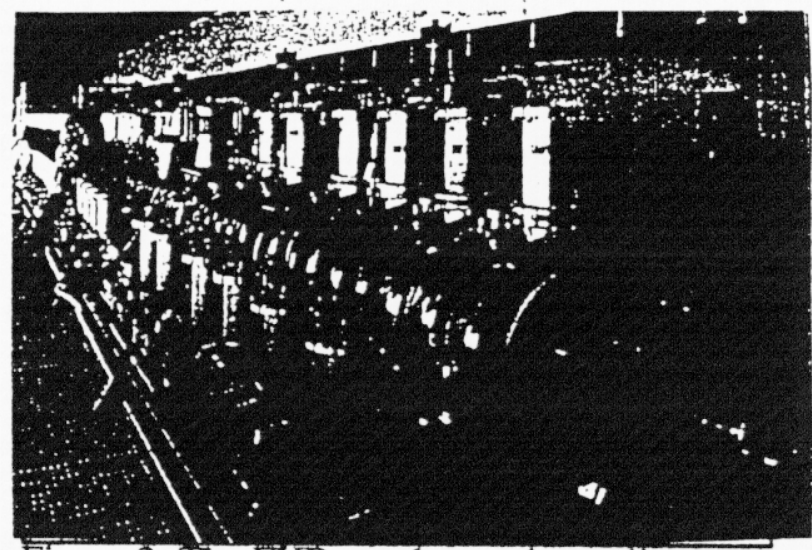

Figure 2: The FXR accelerator beam line as seen from

ector

\section{The FXR Beam Upgrade Effort}

The five year FXR Beam Upgrade ${ }^{3}$ effort, which was completed in mid 1996, addressed,

1) the installation of sixty-two improved focus coils used for beam transport and the addition of steering coils with each focus coil,

2) the redesign of the electron injector to increase both the exiting electron beam energy and current,

3) modifications to the basic accelerator cell to reduce the coupling between the electron beam and the radio frequency resonant modes of the accelerator cell, and

4) the addition of electron beam diagnostics to monitor electron beam current profile and beam centroid location after each four cell block. 
Throughout most of this upgrade effort, the accelerator was down for only brief periods of time because installation of upgraded components was conducted in stages which lasted on the average about 2-3 weeks each. Therefore the FXR was available for programmatic use a radiography machine much of the time.

\section{1) Improved focus coils with additional steering coils}

The original FXR focus coils were fabricated using $0.340^{\prime \prime}$ square hollow copper conductor and were crudely wound on wooden mandrels and potted in epoxy. The result was a coil whose mechanical and magnetic axes were different and indeterminate. The coils fit over the $15 \mathrm{~cm} \varnothing$ beam pipe and, upon installation, were shimmed to be coaxial to the cell centerline, with the resulting measured alignment accuracy ranging from 10 to $30 \mathrm{mr}$. tilts and up to $5 \mathrm{~mm}$ displacements from the accelerator axis. In addition, the magnetic centerline of a coil was not straight but 'wandered' due to the variations in the pitch of the windings. Tuning was made difficult because, along with the axial magnetic field, was a transverse field set up by the coils misalignment which tended to steer the electron beam from the axis.

The design of the replacement focus coils represent a substantial improvement to the original design and is discussed Zentler ${ }^{4}$. The cross-section of a focus coil and its location in the accelerator cell is shown in figure 3 .

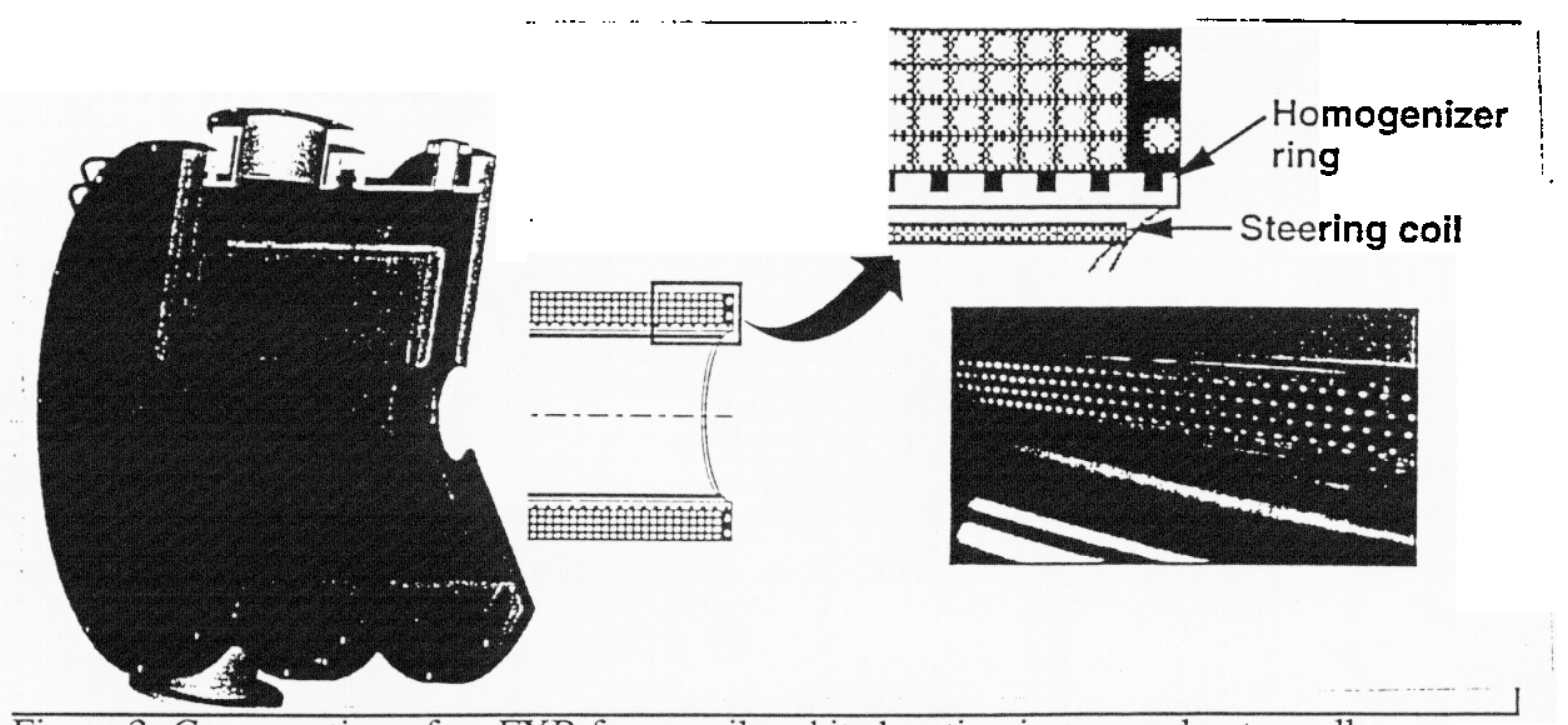

Figure 3: Cross-section of an FXR focus coil and its location in an accelerator cell.

The new solenoids are bifilar wound, unlike the original ones which were 2, two-layer conventional solenoids (wound one inside the other). The advantages of a bifilar design are: the symmetry of the design minimizes the on-axis dipole field errors; less wind pitch variation; and the shorter cooling channels which permit higher current densities and hence increased magnetic field strength. The coils use 0.289 " square hollow copper conductor and generate magnetic fields of 5.67 Gauss/ampere with a maximum field of 2500 Gauss, as compared to the original coils having 4 Gauss/ampere with a maximum field of 2000 Gauss.

Each solenoid was wound on a machined aluminum mandrel which allowed for the accurate winding as well as permitted the accurate placement of the solenoid in the accelerator cavity. Grooves, machined in the outside surface of the aluminum mandrel, were wrapped with 
transformer steel ribbon and serve as accurately placed homogenizer rings. The homogenizer rings short out any remaining transverse magnetic fields errors.

Printed circuit steering coils were installed in every solenoid to further reduce the magnetic misalignment and allow for active steering of the electron beam. These coils can generate a maximum of 733 Gauss-cm with an energizing current of 5.0 amperes, with typical operation being less than 25 amperes.

The net result of this effort was a measured tilt and displacement throughout the accelerator beam line of 0.1 to 2 milliradians tilt and displacements of .01 to $.2 \mathrm{~mm}$. This represents a 10 to 20 times reduction of the transverse magnetic field errors typical of the pre-upgrade coils (see Figure 4). The active steering, using the printed circuit steering coils further reduce the amplitude of the corkscrew motion and the resulting spot size at the Bremstrahlung target.

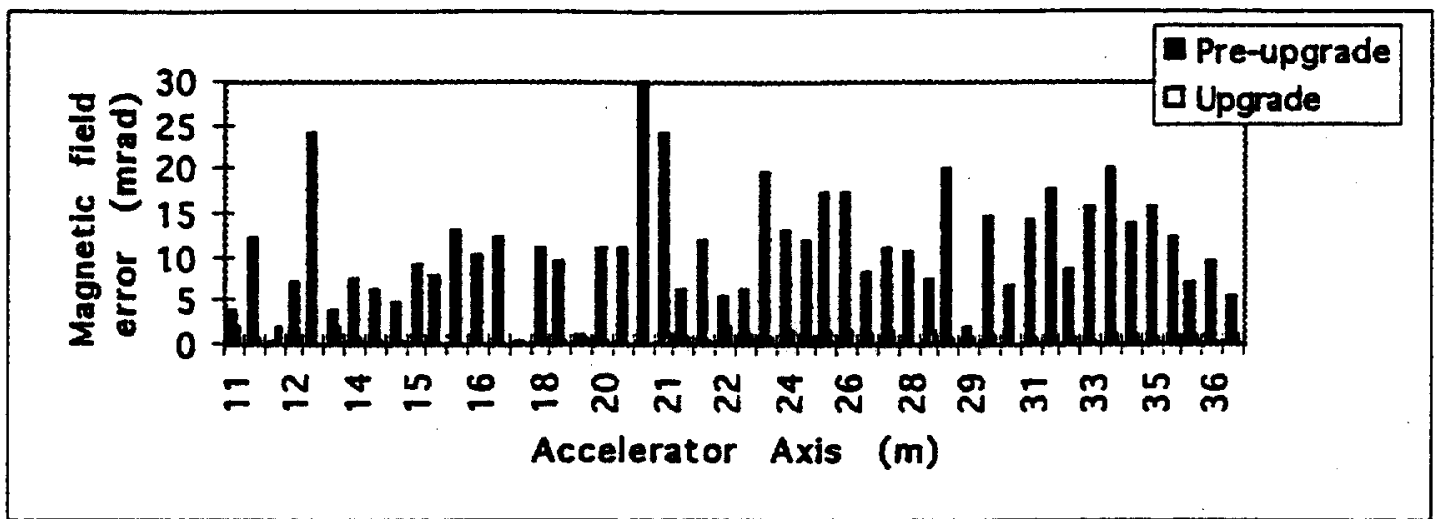

Figure 4. A comparison of magnetic fiedd misalignment prior to and after the installation of the new focus coils. All measurements were made in situ.

In order to minimize accelerator downtime, the FXR accelerator was upgraded with the new focus coils by removing a single 4 unit section, replacing the new coils and then reinstalling it into the accelerator. To accomplish this with a minimum of disruption to FXR operations, a new 4 unit accelerator section, complete with improved cell focus coils, was fabricated. The upgrade procedure consisted of removing an old section and immediately replacing it with the refurbished, upgraded spare. The section removed was then upgraded and refurbished over a 5 week time span, and became the replacement for the next section.

The first cell block exchange occurred in April 1993 and took two weeks to accomplish, at which time the accelerator was up and operational. The accelerator sections in the high energy, or high gamma, end of the accelerator were replaced first. The last cell block was exchanged in November 1995.

\section{2) Redesion of the electron injector}

The FXR electron injector was upgraded to generate a higher current, higher energy and higher quality electron beam. The original injector ${ }^{5}$ consisted of six, near standard accelerator cells. A cathode stalk threaded four of the cellis and added the voltages from those cells, while an anode stalk threaded through the remaining two cells. All six injector cells each operated at $200 \mathrm{kV}$ producing a total voltage across the diode of $1.2 \mathrm{MV}$ and a 2.2 kilo ampere electron beam. The diode used a velvet field emission cathode and a grided anode. 
The upgraded injector was designed to operate using ten accelerator cells with each cell energized to $250 \mathrm{kV}$ for an overall voltage of $2.5 \mathrm{MV}$ and a 3 kilo ampere electron beam (see figure 5). The injector voltage and electron beam current wave forms are shown in figures $6 \mathrm{a}$, b and an enlarged view of the diode and beam envelope through the transition region are shown in figure 7 . This

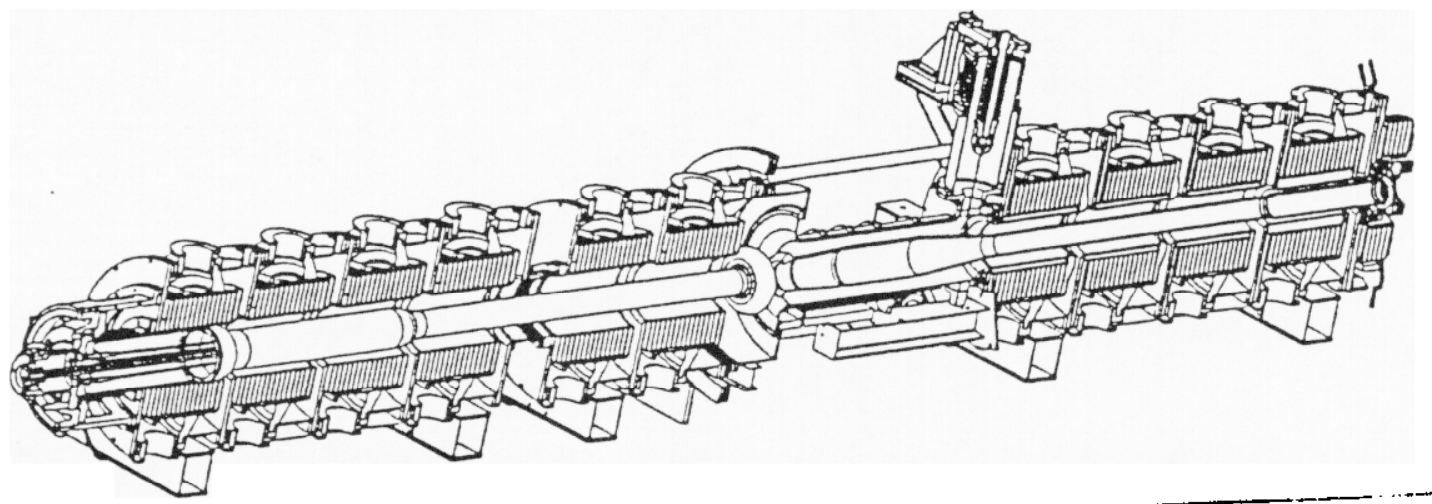

Figure 5:The FXR $2.5 \mathrm{MV}, 3 \mathrm{kA}$ injector consists of 10 slightly modified accelerator cells.

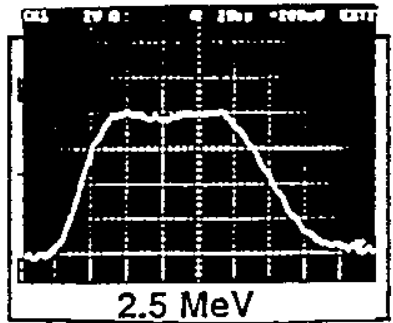

Fig. 6a: Injector Voltage

Wave form. $20 \mathrm{~ns} /$ div.

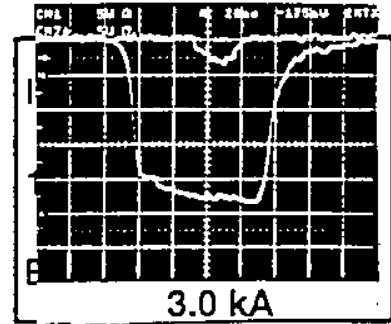

Fig. 6b: Injector Current

Wave form. $20 \mathrm{~ns} / \mathrm{div}$.

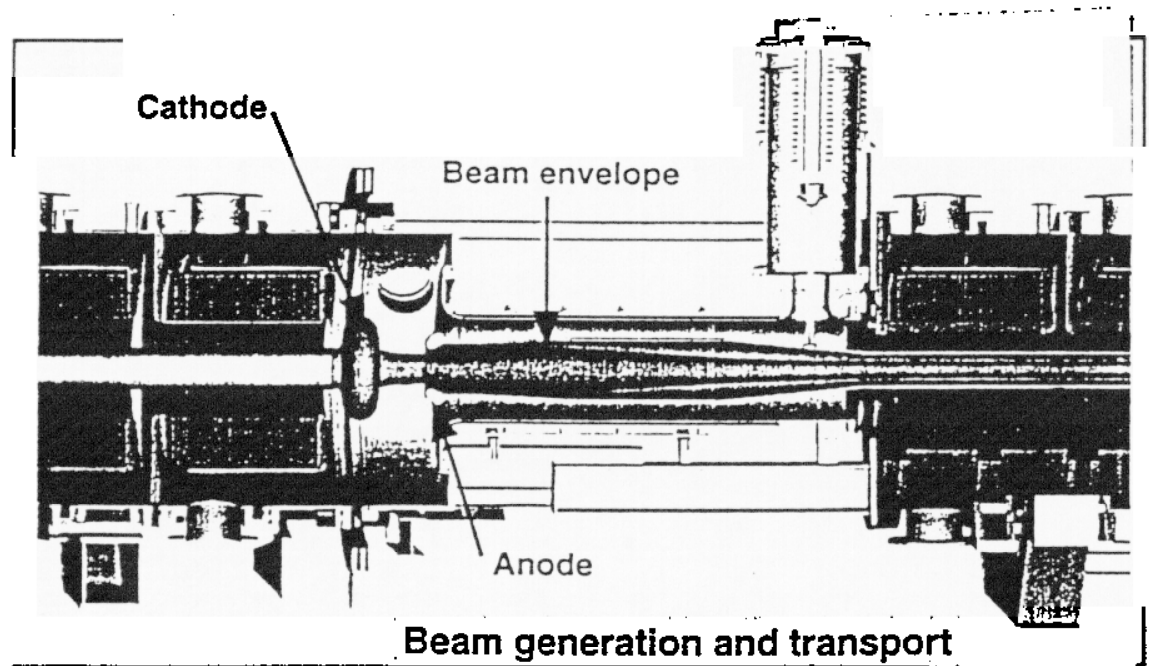

Figure 7: Enlarged view of the injector diode and electron beam envelope.

effort represents a dramatic upgrade to the existing injector and involves a redesign and replacement of the diode, the anode and cathode stalks, and the transition region. An additional nine coils are required for the injector, representing five separate solenoid designs. Each of the new focus coils were based upon the accelerator focus coil design and incorporated homogenizer rings and steering coils. The increased beam energy and the removal of the anode mesh results in a lower emittance (higher brightness) electron beam than exists with the original injector. A beam of lower 
emittance relates to a smaller spot size on target. Like the original injector, the upgraded injector uses a velvet field emission cathode and, unlike the present injector, a gridless anode.

\section{3) Reducing the EM coupling between the electron beam and resonant electromagnetic modes of the accelerator cell}

The Beam Breakup Instability, or BBU, makes a contribution to the overall electron beam centroid motion and final beam spot size on the target. BBU is caused by the interaction of the electron beam with the accelerator cell natural electromagnetic resonances. An off axis beam couples to a non axi-symmetric RF cavity mode, which then imparts a transverse force to the electron beam, kicking it further off axis. Theoretical models, computer simulations and experimental results all identify this to be a much more significant problem as the transported electron beam current is increased from 2 to 3 kilo ampere. Computer modeling ${ }^{6}$ of the RF response of the accelerator cavity using AMOS FDTD simulation code, along with experiments conducted on an actual accelerator cell have identified modifications that significantly reduce the cell RF resonance at 820 MHz. The changes include adding a thin walled RF reflector at the outer edge of the accelerator gap and placing RF absorbing ferrite torroids in the cavity feed and load lines.

\section{4) Electron beam diagnostics}

Beam bugs were placed at 16 locations along the beam line. They are resistive wall type monitors described by Struve ${ }^{7}$ which provide time resolved beam current and the beam centroid location. A bank of $200 \mathrm{MHz}$ digitizers allow all beam bug signals to be acquired on a single shot. The beam bug signals are used in conjunction with the 'Tuning $\mathrm{V}^{\mathrm{V}}$ algorithm developed by $\mathrm{Chen}^{8}$ and typical results are discussed in the paper by ${ }^{\circ}{ }^{9}{ }^{9}$ in these proceedings. The 'Tuning $\mathrm{V}^{\prime}$ ' involves adjusting each steering coil, to minimize the beam centroid motion. This process starts at the beginning of the accelerator and works through to the end. This method has proven to be very effective in reducing the beam corkscrew motion.

\section{Experimental results}

The FXR Upgrade Project has significantly enhanced the radiographic capabilities of LLNL's hydrotest facilities (see Table 1). Analysis of the $1.9 \mathrm{~mm}$ spot shows that a central hot spot of 1.1 $\mathrm{mm}$ in diameter exists with tails that degrade it to the larger diameter. A dose of 100-110 Rads @ one meter is gotten when a $0.8 \mathrm{~mm}$ diameter $\mathrm{x}$-ray collimater is positioned in front of the target resulting in a spot size of $0.93 \mathrm{~mm}$ and a dose/(dia.) ${ }^{2}$ of 127 . As time permits in the hydrotesting schedule, tuning of the FXR continues with the goal of further increasing the transported beam current while at the same time reducing the electron spot size at the target, and hence the $x$-ray spot size. Tunes that employ higher magnetic fields are being tried to further increase the transported

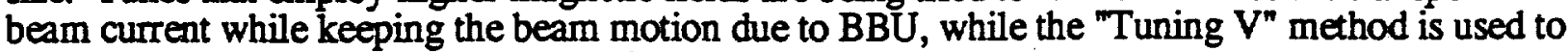
reduce the beam overall corkscrew motion.

Table 1: FXR Operational Capabilities

\begin{tabular}{|lll|}
\hline & Pre-Upgrade & Work in progress \\
Injector energy & $1.2 \mathrm{MeV}$ & $2.5 \mathrm{MeV}$ \\
Final beam energy & $16 \mathrm{MeV}$ & $16-18 \mathrm{MeV}$ \\
Beam current & $2.2 \mathrm{kA}$ & $2.3-3.4 \mathrm{kA}$ \\
X-ray dose (@ 1 meter) & $300 \mathrm{Rad}$ & $325-550 \mathrm{Rad}$ \\
Spot size (Gaussian FWHM) & $2.2 \mathrm{~mm}$ & $1.9 \mathrm{~mm}$ \\
X-ray dose/(dia) & 69 & 127 \\
\hline
\end{tabular}


The. FXR Double Pulse Upgrade

A second upgrade, the Double Pulse Upgrade, began in 1996 and will make the FXR capable of producing two on-axis radiographs of a single device at a time interval of from 1 to 5 microseconds and will allow researchers to follow the time evolution of the late-time details of an implosion.

\section{References}

1 The FXR Accelerator - Kulke,Et al 1981Particle Accelerator Conf

2 The FXR E\&TR, Internal LLNL Publication

3 FXR Beam Upgrade Project Plan

4 The FXR coil design

5 Injector design/optics PAC 1981

6 Scott Nelson's work

7 Beam Bugs, Struve

8 The Tuning V method

9 Ong, this conference 


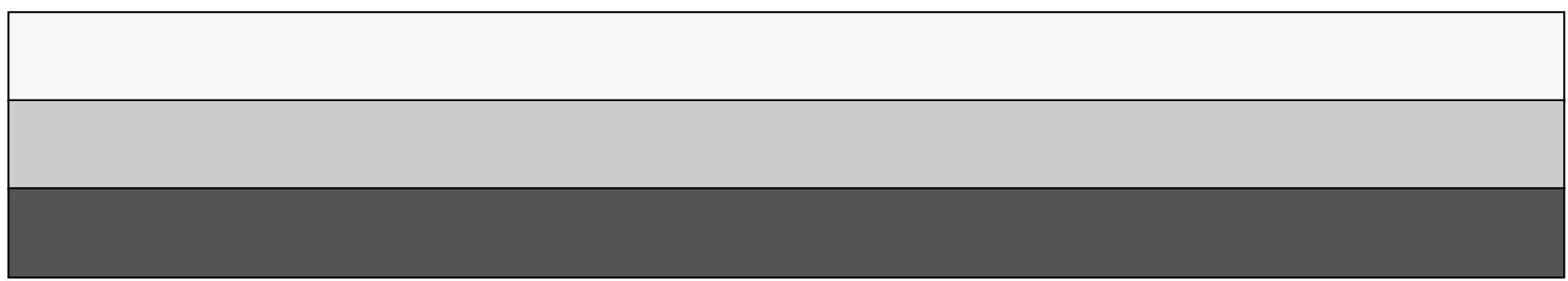

